\title{
Mars Radiation Risk Assessment and Shielding Design for Long-Term Exposure to Ionizing Space Radiation
}

\author{
Ram K. Tripathi \\ NASA Langley Research Center \\ Hampton VA 23681 \\ 757-864-1467 \\ ram.k.tripathi@nasa.gov \\ John E. Nealy \\ NASA Langley Research Center \\ Hampton VA 23681 \\ 757-864 -8094 \\ john.e.nealy@nasa.gov
}

\begin{abstract}
NASA is now focused on the agency's vision for space exploration encompassing a broad range of human and robotic missions including missions to Moon, Mars and beyond. As a result, there is a focus on long duration space missions. NASA is committed to the safety of the missions and the crew, and there is an overwhelming emphasis on the reliability issues for space missions and the habitat. The cost-effective design of the spacecraft demands a very stringent requirement on the optimization process. Exposure from the hazards of severe space radiation in deep space and/or long duration missions is a critical design constraint and a potential 'show stopper.' Thus, protection from the hazards of severe space radiation is of paramount importance to the agency's vision. It is envisioned to have long duration human presence on the Moon for deep space exploration. The exposures from ionizing radiation galactic cosmic radiation and solar particle events - and optimized shield design for a swing-by and a long duration Mars mission have been investigated. It is found that the technology of today is inadequate for safe human missions to Mars, and revolutionary technologies need to be developed for long duration and/or deep space missions. The study will provide a guideline for radiation exposure and protection for long duration missions and career astronauts and their safety. ${ }^{12}$
\end{abstract}

\section{TABLE OF CONTENTS}

1. INTRODUCTION...............................1

2. SHIELD OPTIMIZATION.........................2

3. SPACE ENVIRONMENT...........................4

4. RELIABILITY SHIELD DESIGN.................6

5. MARS MISSION ANALYSIS......................7

6. CONCLUSIONS.............................8

REFERENCES...............................8

BIOGRAPHY.................................9

$1-$
${ }^{1}$ 1-4244-1488-1/08/\$25.00 @2008 IEEE.
${ }^{2}$ IEEEAC paper \#1291, Version 4, Updated November 23, 2007

\section{INTRODUCTION}

An enabling technology for the exploration, the development, and the commercialization of space is a costeffective means of reducing the health risks from exposures to galactic cosmic rays (GCR) and a possible solar particle event (SPE). This has been a well-recognized challenge and a critical enabling technology for exploration in which astronaut health effects are of principal concern. Even more so with the development of space infrastructure and the eventual commercialization of space as new materials and other space products are identified and as larger numbers of civilians become involved in space based careers. At the present stage of space exploration, the astronaut corps is a select group of individuals who normally enter into service near mid-life and have a very limited career duration that allows unusually high annual exposures during their short career. Even then the mitigation of health risks is a great challenge. As we begin to build infrastructure for commercialization, the involvement of more ordinary career workers who will live and work in space will require a reassessment of allowable exposure limits and undoubtedly a substantial reduction in allowable annual exposure. Even more challenging is the "personal family explorer" who may choose to have a family vacation in space. The use of shielding to control exposure and the role of pharmacology in risk mitigation are critical issues in space development.

Radiation health-risk mitigation includes advanced propulsion systems, crew selection, biological countermeasures, and operational approaches. Fundamental to risk mitigation is the advance in knowledge of the radiation risk coefficients for which large design margins are presently required. Reducing the risk uncertainty is a cost effective approach using data collection and research to possibly add years to the careers of space workers. It is the purpose of the present development to incorporate our current state of knowledge of radiation risks into the shield design process. Such a development will allow evaluation of radiation risk-uncertainty impact on shield design, allow the design of reliable shielding for future missions, and 
quantify the advantage of future research in terms of strict engineering practice.

In view of the inadequate information about biological response to cosmic radiation and lack of a definitive criterion for acceptable levels of risks for deep space missions, reliability based methods for radiation shielding and protection, as discussed herein, are of enormous importance to human exploration and development of space. Even if a definitive criterion were available for deep space missions, uncertainties in biological response to deep space radiations would remain with us for a long time. Hence, the reliability based methods for the design process for radiation protection and shielding will continue to play a significant role for space exploration for many years to come.

The National Council on Radiation Protection and Measurements (NCRP) (1989) [1] suggested that the low earth orbit (LEO) exposure limits be used as a guideline for deep space mission studies. Recently, NCRP (2006) [2] has made recommendations for space missions beyond LEO. Although the basic physics of shield evaluation has made great progress [3], the primary uncertainty in shielding estimates result from the large uncertainty in biological response to high charge and energy (HZE) ions of the galactic cosmic rays. In the present study, we will begin the development of a software framework to evaluate the best solution to shielding problems under the limitations of biological uncertainty. In essence, the distribution of probable biological response (related to uncertainty) will be used to evaluate the phase-space of probable shielding solutions. The best shield solution corresponding to a specified confidence level of biological risks will provide a means of entering this uncertainty into the design process. Thus, design reliability is assured within current knowledge by robust design processes that can be specified to a given level of reliability. To make such methods practical, each probable design needs to be optimized with respect to onboard materials and across-the-mission architecture. Optimization frameworks are being developed and preliminary analysis of probabilistic reliability based methods is the focus of the present paper.

The development of reliability-based methods is dependent on shield analysis software and the ability to calculate the quantities required for reliability design processes and further enable design optimization across mission architectures. An enabling technology to accomplish such tasks is the development of highly efficient deterministic computational procedures for shield evaluation able to provide high-quality estimates of the improbable events so important to biological injury. At a practical level, this means evaluation of radiation field quantities within complex structures within minutes to enable optimization procedures.
In the present paper, we will first review the underlying quantities to be considered and their implementation into the design process. We will then use this development to examine a Mars reference, Mars/Venus swing by mission and evaluate the impact of probabilistic reliability design methods compared to nominal methods suggested by the NCRP (1989) [1]. In this application, we will consider an 860-day Mars mission and a 365-day Mars/Venus swing by mission. Clearly, future developments will require a more complex mission scenario and optimization across a more complex array of habitats and vehicles. We will then draw conclusions about shielding requirements, using the reliability process for human missions to Mars.

\section{SHIELD OPTIMIZATION}

Shield mass can be a high cost factor in system designs for the long-term operations required and optimization methods in the design process will be critical to cost-effective progress in space development [3]. Limiting the time of transfer to duty station or the mission time within the solar cycle as well as the choice of materials used in construction can reduce the shield mass required on specific missions [4]. Unfortunately, adequate optimization procedures have not been available to minimize the mass and the associated costs for a given mission scenario.

In the past, an amount of exposure was assigned to each mission segment and developed as a subjective strategy with relative improvements of costs through material trades dependent on off-optimum design solutions. It is the purpose of the present study to develop the necessary optimization methods for minimum mass determinations to be used in performing trade studies to enable objective trade reduction costs since strategies for meeting exposure constraints are optimized over the entire mission architecture for each trade. In addition to optimized design trades, we will also consider the implementation of the principle of as low as reasonably achievable (ALARA) required by federal regulation and normally ignored in mission design studies. The ALARA principle will be met by added protection of the crew quarters where members will spend a significant fraction of each day sleeping. The main crew quarter design will also be used as the shelter from potential solar particle events during the mission. In this respect, we assume that an adequate strategy for exposure limitation during extra vehicular activity (EVA) is available and the design is mainly the habitable volume and crew quarter/SPE shelter. Emergency planning in the case of an accidental SPE exposure will have to be part of the overall mission plan and is not considered in the present study.

\section{Exposure and other Constraints}

The basic requirement on astronaut exposure limitations established by the NCRP is that radiation induced excess fatal cancer risks from career exposures are to be limited to 
be less than 3 percent and early radiation syndrome (nausea, vomiting...) is to be avoided [1, 5-7]. Qualitatively, this is similar to the requirements for terrestrial radiation workers. The radiation environment is of such a character that career radiation exposure limits have been given by the NCRP in terms of a local tissue related quantity known as dose equivalent (Sievert) [1]) given by,

$$
H=\int Q(L) D_{L} d L
$$

where Q (L) is the quality factor (International Commission of Radiological Protection(ICRP 1991)) [8] relating to the difference in induced risk of differing particle types delivering the same dose and $\mathrm{D}_{\mathrm{L}}$ is the dose (Gray) from components with linear energy transfer between $\mathrm{L}$ and $\mathrm{L}+$ dL. Note that Eq. (1) breaks the convention of the ICRP (1991) [8] who have recommended radiation field weighting factors for estimation of fatal cancer risks, which does not depend on the local tissue field. The argument given by the ICRP is that the uncertainty introduced through such a nonlocal approximation is indicative of the uncertainty in risk estimation methods in distinction to Eq. (1) that gives the appearance of a quantified risk. The approach by the NCRP in recommending Eq. (1) allows a quantitative treatment of uncertainty as noted in reference [3] and enables the development of reliability based methods, as we will show herein.

The dose equivalent quality factor was developed using cell death and mutation data largely from animal experiments to model stochastic effects and was not ideal for modeling deterministic effects. Thus, the quantity gray equivalent was introduced in NCRP report no. 132 [9] and defined in greater detail in NCRP report no. 142 [10]. Gray equivalent, $G_{T}$, for tissue type $T$ is defined in NCRP report no. 142 as

$$
G_{T}=\sum_{i} R_{i} D_{T, i}
$$

where $D_{T, i}$ is the mean absorbed dose to tissue $T$ and $R_{i}$ is the recommended relative biological effectiveness number. The relative biological effectiveness (RBE) numbers defined in NCRP report no. 132 [9] are given in Table 1.

Table1. Recommendation relative biological effective

\begin{tabular}{|c|c|}
\hline \multicolumn{1}{|c|}{ numbers } & RBE \\
\hline 1 to $5 \mathrm{MeV}$ neutrons & 6.0 \\
\hline 5 to $50 \mathrm{MeV}$ neutrons & 3.5 \\
\hline $\begin{array}{c}\text { Heavy ions (e.g. helium, carbon, neon, } \\
\text { argon) }\end{array}$ & 2.5 \\
\hline Protons $>2 \mathrm{MeV}$ & 1.5 \\
\hline
\end{tabular}

It should be noted that the relative biological effectiveness numbers are to be applied to the ambient radiation fields external to the human body but inside the vehicle and/or habitat.

NCRP report no. 132 also defined the quality factor effective dose, $E$, as a better measure of whole body exposure [11] than dose equivalent to blood forming organs, BFO. Effective dose is a weighted average of dose equivalent to various organ and/or tissue types and is defined by Eq. (3) as

$$
E=\Sigma w_{T} H_{T}
$$

where $H_{T}$ is the average dose equivalent to tissue type $T$ and $w_{T}$ is the weighting factor for tissue type $T$. The effective dose weighting factors designated in NCRP report no. 132 are given in Table 2.

Table 2. Effective dose weighting factors, wt

\begin{tabular}{|l|l|l|l|}
\hline $\mathbf{0 . 0 1}$ & $\mathbf{0 . 0 5}$ & $\mathbf{0 . 1 2}$ & $\mathbf{0 . 2 0}$ \\
\hline Bone surface & Bladder & Bone marrow & Gonads \\
\hline skin & Breast & Colon & \\
\hline & Liver & Lung & \\
\hline & Esophagus & Stomach & \\
\hline & Thyroid & & \\
\hline & Remainder & & \\
\hline
\end{tabular}

Here the "Remainder" tissues refer to adrenals, brain, small intestines, large intestines, kidney, muscle, pancreas, spleen, thymus, and uterus.

Permissible radiation exposure limits (PEL) are being set up for LEO. Current limits for LEO operations limits were defined in NCRP report no. 98 [1] and adopted by NASA. These limits are shown in Tables 3 and 4.

Table 3. LEO exposure limits for blood forming organs, eyes, and skin for all ages (NCRP 98).

\begin{tabular}{|l|c|c|c|}
\hline & $\begin{array}{c}\text { BFO } \\
(\mathrm{Sv})\end{array}$ & $\begin{array}{c}\text { Eye } \\
(\mathrm{Sv})\end{array}$ & $\begin{array}{c}\text { Skin } \\
(\mathrm{Sv})\end{array}$ \\
\hline Career & $\begin{array}{c}\text { Table } \\
4\end{array}$ & 4.00 & 6.00 \\
\hline Annual & 0.50 & 2.00 & 3.00 \\
\hline 30 Day & 0.25 & 1.00 & 1.50 \\
\hline
\end{tabular}

Table 4. LEO career BFO dose equivalent limits (Sv) (NCRP 98)

\begin{tabular}{|c|c|c|c|c|}
\hline Age & 25 & 35 & 45 & 55 \\
\hline Male & 1.5 & 2.5 & 3.2 & 4.0 \\
\hline Female & 1.0 & 1.75 & 2.5 & 3.0 \\
\hline
\end{tabular}

It should be noted here that the career limits in Table 4 are based on a 3\% excess lifetime fatal cancer risk. This means that the astronaut's risk of developing a fatal cancer during his or her lifetime is increased by no more than 3\%. Also, note that in addition to the limits defined in Tables 3 and 4 , 
NASA is required to follow the principle of ALARA, keeping exposure "As Low As Reasonably Achievable.”

In NCRP report no. 132, new exposure limits incorporating the new dosimetric quantities, gray equivalent and effect dose, were recommended. These limits are shown in Tables 5 and 6.

Table 5. LEO exposure limits for blood forming organs, eyes, and skin for all ages (NCRP 132)

\begin{tabular}{|l|l|l|l|}
\hline & $\begin{array}{l}\text { BFO } \\
(\text { Gy-Eq) }\end{array}$ & $\begin{array}{l}\text { Eye } \\
\text { (Gy-Eq) }\end{array}$ & $\begin{array}{l}\text { Skin } \\
(\text { Gy-Eq) }\end{array}$ \\
\hline Career & Table 6 & 4.00 & 6.00 \\
\hline Annual & 0.50 & 2.00 & 3.00 \\
\hline 30 Day & 0.25 & 1.00 & 1.50 \\
\hline
\end{tabular}

Table 6. LEO career whole body effective dose limits (Sv) (NCRP 132

\begin{tabular}{|l|l|l|l|l|}
\hline Age & 25 & 35 & 45 & 55 \\
\hline Male & 0.7 & 1.0 & 1.5 & 3.0 \\
\hline Female & 0.4 & 0.6 & 0.9 & 1.7 \\
\hline
\end{tabular}

Here, also, the career limits are based on a 3\% excess lifetime fatal cancer risk.

Aside from the radiation health risks, the psychological well-being and its impact on crew performance also affects the shield design [4]. Crew performance level is related in part to the length of the mission and the volume of the work/living areas of the spacecraft. The design performance levels of Optimal, Performance Limit, and Tolerable are essential inputs to design processes [4]. Rather small volumes are useful over short time periods but long missions require sufficient space for a crew to perform at reasonable levels. In this study, we use the optimal design for the habitable volume and the Tolerable design for the crew quarters which also serves as the SPE shelter.

In addition to the trapped radiations and the GCR able to penetrate the geomagnetic field to LEO, there are occasional solar particle events able to penetrate the geomagnetic field. The solar particle source is mainly composed of protons of similar quality as the trapped protons and the limitations in Tables 5 and 6 are applicable. The implications of the galactic cosmic ray exposures on LEO operations have not been fully evaluated with respect to exposure limitations.

Beyond the geomagnetosphere, the GCR are the dominant long-term exposure hazard. Generally for deep space operations, the provision of protection from galactic cosmic rays will provide most, if not all, the protection required from solar particle events. The GCR are ions of every known element with spectra spanning rather modest energies to very high energies. Although cosmic rays are mostly protons and helium ions, the high-energy heavy ions or HZE ions deliver a large fraction of the dose equivalent.
The LET of a given ion is proportional to the square of the charge and a very broad range of LET is encountered beyond LEO for which there is little biological data.

As given in Eq. (1), the cancer risk is related to the LET distribution of the dose modified by the quality factor Q (L). Control of cancer risk is accordingly to control the LET distribution of particles, and in this not all materials are equal. The LET is dependent on ion type and energy that is modified by fragmenting the ions in nuclear processes to different ion types and slowed in speed by atomic interactions. Both processes depend on the composition of the material. A third related process is the generation of new particles by collision with the complex nuclei in the shield material. One must balance these three processes in the selection of material with which to construct the shielding to protect the astronauts.

The most efficient shielding material is provided by liquid hydrogen. It shows the greatest attenuation of high LET components with minimal addition of troublesome secondary radiations. Also relatively efficient is water, and aluminum has been shown to be a poor material for spacecraft construction since secondary radiations create an additional hazard and any improved protection occurs only at very large depths. Materials with even higher atomic numbers than aluminum $(Z=13)$ are progressively more hazardous. Even local in situ materials need to be used with care because of the nature of the secondary radiations produced. The augmentation of local materials with polymer binders can help in controlling secondary radiations but requires more development, testing, and complete evaluation for the specific application. Some of the required tools for this evaluation are the subject of the present paper.

\section{SPACE ENVIRONMENT AND SHIELDING MATERIALS}

In order to quantify radiation exposure in space, it is required that the external ambient ionizing radiation environment be specified in terms of individual constituents and their respective energy fluxes. A great quantity of observational space environmental data from instrumented space platforms has been amassed in recent decades and used in developing computer models serving to define, as well as possible, the composition and temporal behavior of the space environment $[12,13]$. From the standpoint of radiation protection for humans in interplanetary space, the heavy ions (atomic nuclei with all electrons removed) of the GCR and the sporadic production of energetic protons from large SPEs must be dealt with. The GCR environmental model used herein is based on a current version in which ion spectra are modulated between solar maxima and minima according to terrestrial neutron monitor data assuming the radial dependent diffusion model of Badhwar et al. [12]. The modeled spectra for solar minimum in 1977 
and solar maximum in 1990 as given by Badhwar are shown in Fig.1. There are only a few percent differences between the environments measured near Earth and what is observed near Mars and other locations near the Earth's orbit about the sun. These anticipated differences are less than the model uncertainty and will be ignored in the present study.

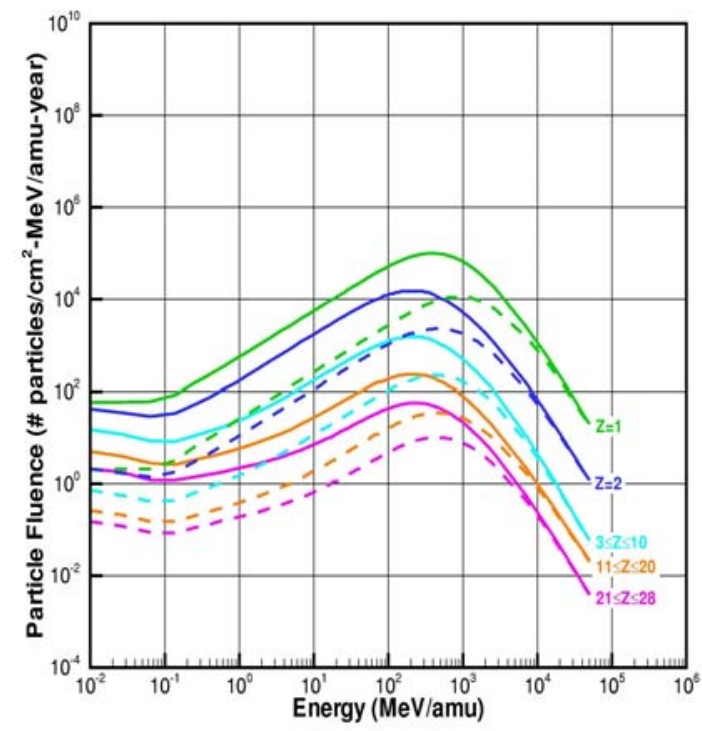

Figure 1 - Galactic cosmic ray spectra at the 1997 solar minimum (solid lines) and 1990 solar maximum (dashed lines) according to Badhwar et al.

The environment near a large celestial body is modified by interaction with local materials producing an induced environment and shielding within the subtended angle of such a large body. The surface exposure on a Martian plain is shielded below the horizon but experiences an induced environment (mainly but not exclusively neutrons) produced in the atmosphere and local surface. The Martian surface GCR environment is shown in Fig. 2 at the 1977 solar minimum and the 1990 solar maximum. In addition to the GCR ions and induced environment streaming from overhead, large numbers of neutrons are produced in the Martian surface materials and diffuse from below the surface as shown in Fig. $2(\mathrm{Z}=0)$.

Large SPE have only been observed to occur during times of increased solar activity conditions, and very large energetic events of grave importance to human protection occur only infrequently (avg. 1 or 2 per cycle) and only outside of two years of solar minimum. Among the large events, the largest observed ground level event of the last 60 years of observation is that of February 23, 1956, which produced a 3600 percent increase in neutron monitor levels on the terrestrial surface. The next largest event observed is the September 29, 1989 event with ground level increases of 400 percent or an order of magnitude smaller than that of the Feb. 1956 event. Numerous other ground level events of smaller magnitude have occurred but are about a factor of four lower in magnitude than the Sept. 1989 event. It is known that large SPEs are potentially mission threatening and astronauts in deep space must have access to adequate shelter from such an occurrence. The SPE particle energy spectrum used here has been derived from the event, which took place on September 29, 1989. To provide a baseline worst-case scenario we assume an event of the order of four times larger than that of September 29, 1989, as an event comparable to the August 4, 1972 event from the point of view of space exposure. The September 1989 SPE spectrum is shown in Fig. 3. If we meet 30-day dose rate constraints on an event four times larger than the September 1989 event then it is unlikely that an added factor of two or so larger events (like that of Feb. 23, 1956) would have serious medical consequences. The SPE are likewise altered by the presence of a large body similar to the GCR. The corresponding Martian surface environment is shown in Fig. 4. Neutrons play a relatively more important role on the Martian surface where added neutrons are produced in the overhead atmosphere and the SPE protons are greatly attenuated compared to their effects on lunar surface.

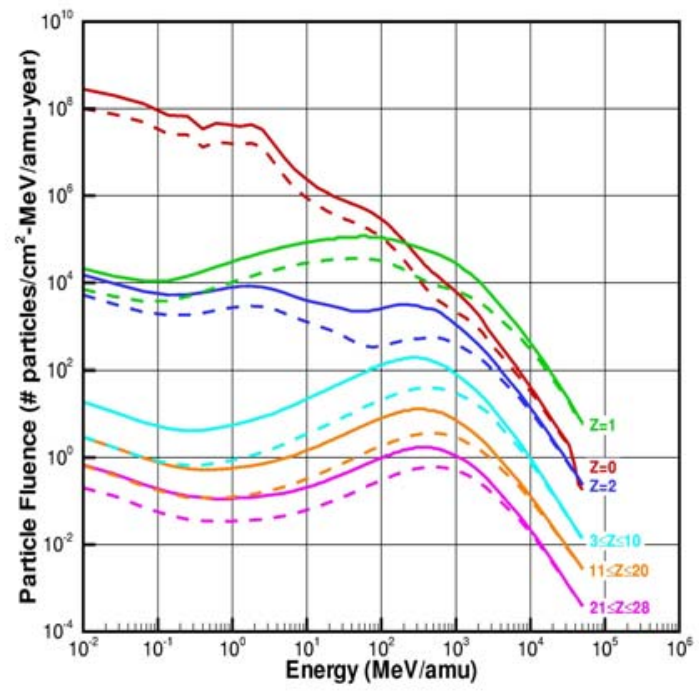

Figure 2 - GCR environment during the 1977 Solar minimum and the 1990 Solar Maximum on the Martian surface (mean altitude) using the Marsgram atmospheric model.

Aluminum is currently used as the base material in shielding requirements. The reason for the selection of remaining materials is that there is increasing hydrogen content in materials as we go down the list. The effectiveness of a given shield material is characterized by the transport of energetic particles within the shield, which is in turn defined by the interactions of the local environmental particles (and in most cases, their secondaries) with the constituent atoms and nuclei of the shield material. These interactions vary 
greatly with different material types. For space radiation shields, materials with high hydrogen content generally have been shown to have greater shielding effectiveness, but often do not possess qualities that lend themselves to the required structural integrity of the space vehicle or habitat. Organic polymers are the exception. The design of properly-shielded spacecraft and habitats for long-duration human presence in interplanetary space will thus require an approach tending toward optimization of a compromise between protective shielding and various other functional aspects of the onboard

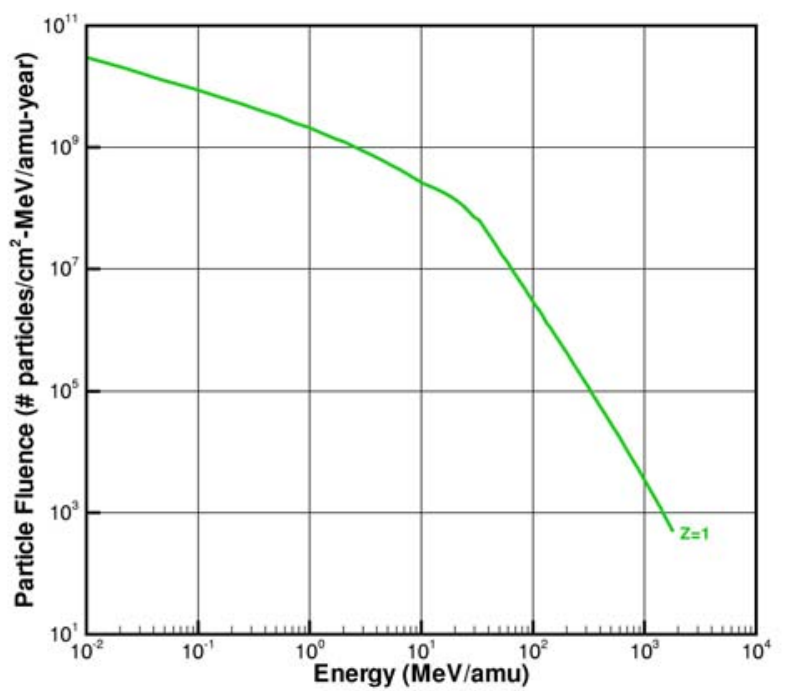

Figure 3 -- SPE spectrum during September 1989 as observed near Earth.

materials. Candidate multifunctional materials for such an optimization approach have been chosen here to represent various contributing elements in a vehicle shield design. Liquid hydrogen and methane are possible fuels that in large quantities may contribute substantially to overall protection. Aluminum has long been a spacecraft material of choice although various forms of polymeric materials show enhanced protection properties such as polyethylene. The polysulfone and polyetherimide are high performance structural polymers. Lithium hydride is a popular shield material for nuclear power reactors, but is generally not useful for other functions. The graphite nanofiber materials heavily impregnated with hydrogen or any composite thereof may well represent a viable multifunctional component in future space structures. In this case study of the graphite nanofiber, hydrogen content is $\sim 68 \%$ wt while in laboratory in single-walled carbon nanotubes (SWNT) hydrogen storage has been achieved $~ 10 \%$ wt. As such, its inclusion here should presently be considered as not yet state-of-the-art and ready for operational studies.

\section{RELIABILITY SHIELD DESIGN}

In application of Eq. (1) in setting exposure limits, the NCRP assumed a linear relation of excess fatal cancer risk to dose equivalent as is appropriate for low dose and low dose rate exposures given by

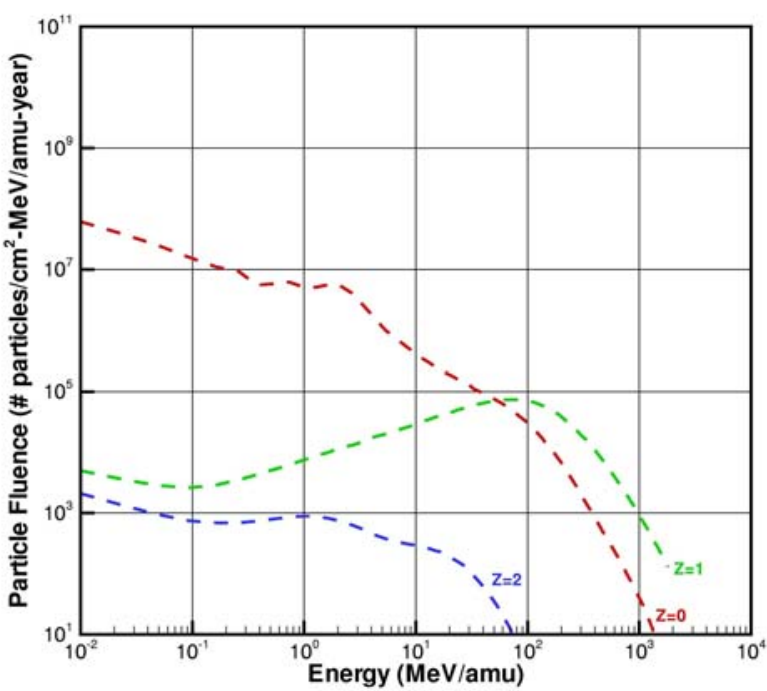

Figure 4 - Martian surface environment during the September 1989 SPE

$$
R(A, G)=R_{0}(A, G) \int D_{L} Q(L) d L
$$

where $R_{0}(A, G)$ is the age $A$ and gender $G$ dependent risk coefficient for the high dose rate exposures in the A-bomb detonations of World War II. The quality factor Q (L) is defined by the ICRP (1991) according to observed RBE factors.

The uncertainty in application of Eq. (4) is discussed in reference [5]. In the present study, we will consider uncertainty in Q (L). Normally in modeling the uncertainty the starting point is Eq. (4) and the nominal values of $\mathrm{Q}(\mathrm{L})$ are replaced $[14,15]$ by stochastic variables $X_{Q}$ as

$$
R(A, G, \kappa)=R_{0}(A, G) \int D_{L} X_{Q} d L
$$

where the $\kappa$ is a statistical variable denoting $R(A, G, \kappa)$ to be a member of a population of possible biological response models associated with the uncertainty in $Q(L)$.

It is desirable to setup the reliability design problem so as to most easily integrate into the mission architecture software derived on the basis of nominal risk coefficients. In that formalism, we used the nominal limits given by the NCRP (2001) based on nominal risk coefficient used in LEO constraints found in Tables 1 and 2. We rewrite Eq. (5) as 


$$
R(A, G, \kappa)=R_{0}(A, G) \int M(L, \kappa) D_{L}(x) d L
$$

where $D_{L}(x)$ is the distribution of dose over LET within a spherical shell of thickness $\mathrm{x}$ assuming that the physics related uncertainties are much less than the biological uncertainties. The function $M(L, \kappa)$ is an off-nominal LET dependent modifying function in which all biological uncertainty resides and is numerically equal to $\mathrm{Q}(\mathrm{L})$ for the nominal risk. In this way, the uncertainty is assigned to the evaluation of "dose equivalent" but is otherwise directly related to the appropriate dose limits. Monte Carlo methodology has been developed [13] for function $M(L, \kappa)$ by comparing relative biological effectiveness to quality factor from available data for mouse tumors, cell transformation or mutations, or cytogenetic endpoints. Our stochastic quality factor $M(L, \kappa)$, where $\kappa$ is a stochastic variable denoting characteristics of the dose equivalent and other quantities and the confidence interval (CI) after 10,000 trials is demonstrated in reference 14 , will be used in future risk assessments. The dose equivalent in this context is given as a stochastic variable by

$$
H(x, \kappa)=\int M(L, \kappa) D_{L}(x) d L
$$

Then the requirement of limiting the lifetime excess fatal cancer risk given by Eq. (6) to less than three percent is equivalent to limiting the value of dose equivalent as given by Eq. (7) to be less than the nominal exposure limits.

In its simplest form the design process can be implemented as follows. For a given shield thickness $\mathrm{x}$, there is a stochastic sequence of associated risks $R(A, G, \kappa)$. The shielding is acceptable to a given confidence level in a confidence interval (CI) when CI percent of the risk sequence lies below three percent. One must seek to find a value of $\mathrm{x}$ that satisfies this requirement. If a mission using this shield configuration was sent then we are CI percent confident that the excess fatal cancer risk to the astronaut would not exceed three percent as a result of the mission. The present formulation is the simplest form of the method and is next to be combined with the mission architecture profile optimization and multidisciplinary optimization procedures.

\section{MARS MISSION ANALYSIS}

Consider a reference and a swing by mission to Mars with a mixed crew of six. The optimum volume of living space is taken as $114 \mathrm{~m}^{3}$ and crew age set at the youngest female. It is assumed that the living space is a right circular cylinder $2.2 \mathrm{~m}$ high. Shield optimization was obtained only for graphite nanofibers and liquid hydrogen (Fig. 5). Again, assuming the walls to be a pressure vessel containing either liquid hydrogen (LH2) or hydrogenated graphite nanofibers with herringbone structure (HGNF), the design mass as a function of age is shown in figure 6. Although this is not the exact geometry and only the shield wall is represented, we see a large impact of uncertainty on the design.

Optimized Mars Reference Mission

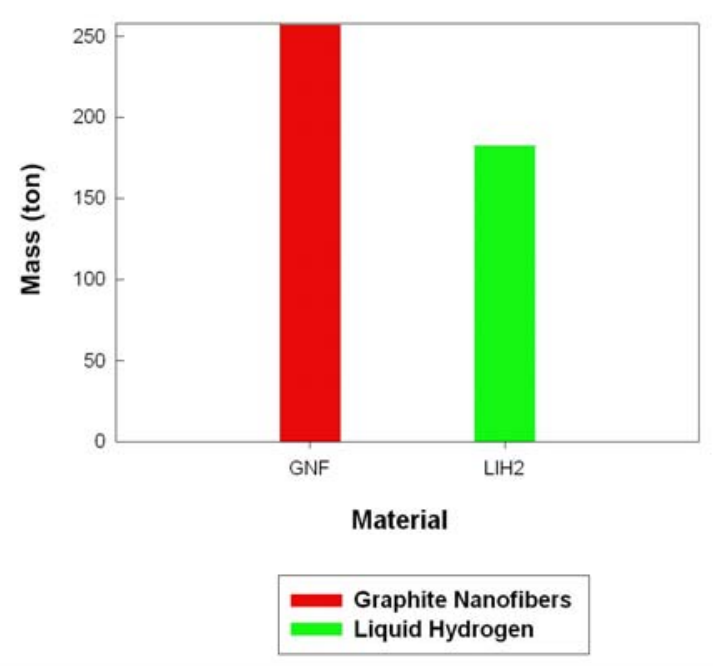

Optimized Mars/Venus Swingby Reference Mission

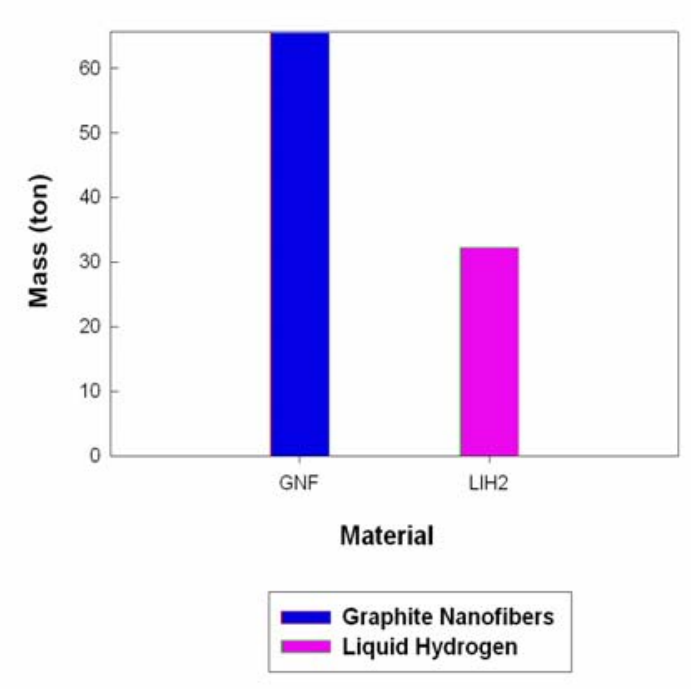

Figure 5 - Shield optimization for two different Mars missions (2yr. upper and 1 yr lower panel)

Optimization was not possible for aluminum and only the LH2 and HGNF showed promise as a shield material for such long duration missions in deep space. As HGNF is a factor of 4 to 6 more efficient at protecting the astronaut than aluminum, it is clear that few material options are available for future deep space Mars missions, and other 
methods such as biological countermeasures will play a pivotal role. At the very minimum, from an engineering design point of view, reliability based methods must be implemented to accurately portray the shielding component of risk mitigation in mission design.
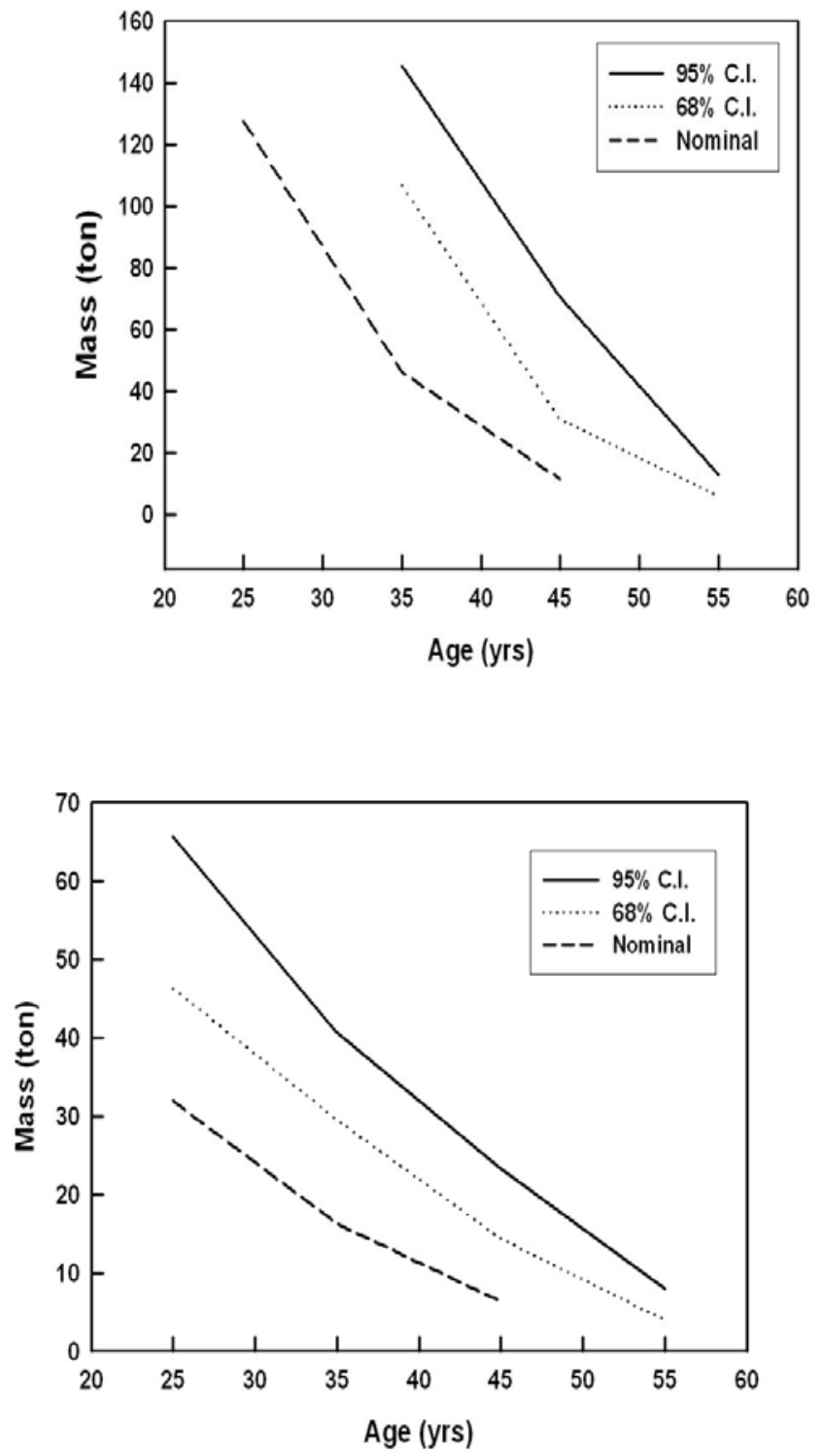

Figure 6 - The Stochastic Design Space for a Two Year Mars Mission for Two Highly Efficient Shield Materials

\section{CONCLUSION}

We have examined an approach to introduce reliability based design methods into shield evaluation and on optimization procedure to assess and control the uncertainties in shield design. Applications to Mars missions display a large impact on the design outcome. Computational procedures based on deterministic solution of the Boltzmann equation are well suited for such procedures allowing optimization processes to be implemented, evaluation of biologically important rare events, and rapid analysis of possible shield optimization outcomes resulting from the biological model uncertainty parameter space. Shield design studies based on nominal biological response models are highly questionable in their result and may lead to designs in which astronaut risk are much higher than anticipated on the basis of such models. Reliability based methods result in designs for which astronaut risk within the limitations of current knowledge is well controlled in the design process. The analysis provides enabling technology for protecting astronauts and missions for long duration and deep space missions. Current technology is adequate for a single lunar mission for casual astronauts. Revolutionary technology needs to be developed for human space missions to Mars for NASA's vision.

\section{REFERENCES}

[1]NCRP, “Guidance on Space Exposures in Space," National Council on Radiation Protection, Bethesda, MD, NCRP Report No. 98 (1989).

[2]NCRP, "Information Needed to Make Radiation Protection Recommendations for Space Missions beyond Low-earth Orbit,” Bethesda, MD, NCRP Report No. 153 (2006)

[3]Wilson, J. W., J.L. Shinn, J.L., Tripathi, R.K., Singleterry, R.C., Clowdsley, M.S, Thibeault, S. A.,. Cheatwood, F.M., Schimmerling, W., Cucinotta, F.A., Badhwar, G.D.,. Noor, A.K., Kim, M.Y., Badavi, F.F., Heinbockel, J.H., Miller, J., Zeitlin, C. and Heilbronn, L., "Issues in Deep Space Radiation Protection,” Acta Astronautica 49, 289-312 (2001).

[4]Tripathi, R.K., Wilson, J.W., Cucinotta, F.A.,. Nealy, J.E, Clowdsley, M.S. and. Kim, M.Y., “Deep Space Mission Shielding Optimization,” SAE 2001-01-2326 (2001).

[5]NCRP, “Uncertainties in Fatal Cancer Risk Estimates Used in Radiation Protection,” NCRP Report No. 126 (1997).

[6]Wilson, J.W., Miller, J., Konradi, A. and. Cucinotta, F.A., "Shielding Strategies for Human Space Exploration," NASA CP-3360 (1997).

[7]Cucinotta, F.A., Schimmerling W., Wilson, J.W., Peterson, L.E., Badhwar, G.D., Saganti, P. and. Dicello, J.F., "Space Radiation Cancer Risks and Uncertainties for Mars Missions,” Johnson Space Center, JSC- 2925 (2001).

[8]ICRP, "The 1990 Recommendations of the International Commission for Radiological Protection,” ICRP Report 60, Pergamon Press, Oxford, UK, (1991). 
[9]NCRP, "Radiation Protection Guidance for Activities in Low-Earth Orbit," National Council on Radiation Protection, Bethesda, NCRP Report No. 132, (2001).

[10]“Operational Radiation Safety Program for Astronauts in Low-Earth Orbit: A Basic Framework,” NCRP Report No. 142, National Council on Radiation Protection and Measurements, Bethesda, MD, 2002.

[11]Clowdsley, M.C., Nealy, J.E., Atwell, W., Anderson, B M., Luetke, N. J and Wilson, J.W., "Calculation of Radiation Protection Quantities and Analysis of Astronaut Orientation Dependence,” AIAA Space 2006.

[12]Badhwar, G.D. and O’Neill, P.M., “Improved Model of Galactic Cosmic Radiation for Space Exploration Eission,” Nucl. Tracks \& Radiat., 20, 403-410, 1992.

[13]Badhwar G.D., et. al, "Inter-comparison of Radiation Measurements on STS-63,” Radiat. Meas. 26; 147-158; 1997.

[14]Tripathi, R.K., Wilson J.W. and Joshi, R.P., "Risk Assessment and Shielding Design for Long-Term Exposure to Ionizing Radiation,” SAE-2006-01-2108, 2005.

[15]Cucinotta, F.A., Kim, M.Y. and Ren, L., "Managing Lunar and Mars Mission Radiation Risks Part I: Cancer Risks, Uncertainties and Shielding Effectiveness," NASA/TP-2005-213164.

\section{BIOGRAPHY}

Ram Tripathi is a Senior Research Scientist at NASA

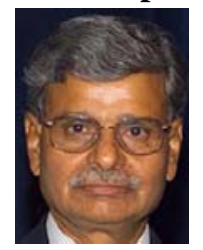
Langley Research Centre and works on radiation protection and shielding $\mathrm{He}$ received his $\mathrm{Ph}$. $\mathrm{D}$. in physics from the University of Kansas and has held professorships, technical leaderships and management positions in physics in various countries including USA, Western Europe (Germany, Great Britain and Belgium) and India. $\mathrm{He}$ has proposed established and developed several new theories such as the existence of Bose condensation of nuclei, unusual states of matter, density dependence of nuclear forces, supernova bounce, in various areas in physics - nuclear physics, radiation physics, atomic and molecular physics, and condensed matter physics. He has wide and extensive experience of work on the development of databases, models for heavy ion interaction with materials and their integration in transport code and has developed state-of-the art methodologies for space radiation risk assessment and protection for NASA's space missions. He is a fellow of the American Physical Society.

John Nealy is a Research Professor at the Old Dominion

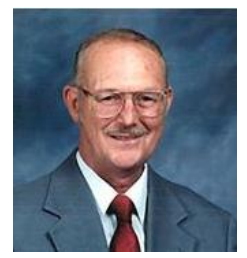

University and works on radiation protection and shielding. Before joining the Old Dominion University, he was a Senior Research Scientist at NASA Langley Research Centre and worked and managed several areas/programs (e.g. space radiation and atmospheric science, CERES and ERBE) of need for space missions including radiation protection and shielding. He received several prestigious awards for his outstanding accomplishments from NASA. Amongst other things, currently, he is working on electron transport and radiation space environment and radiation dose studies. 